\title{
Correção de doenças da aorta torácica com utilização de hipotermia profunda e parada circulatória
}

Bayard GONTIJO FILHO*, Fernando Antônio FANTINI*, Juscelino Teixeira BARBOSA*, João Alfredo de Paula e SILVA*, Marcelo Frederique de CASTRO, Eduardo O. A. PEREDO, Adelso A. PEDROSA, Flávio Donizete GONÇALVES, Mário Osvaldo VRANDECIC*

GONTIJO FILHO, B.; FANTINI, F. A.; BARBOSA, J. T.; SILVA, J. A. P.; CASTRO, M. F.; PEREDO, E. O. A.; PEDROSA, A. A.; GONÇALVES, F. D.; VRANDECIC, M. O. - Correção de doenças da aorta torácica com utilização de hipotermia profunda e parada circulatória. Rev. Bras. Cir. Cardiovasc., 6(1): 11-16, 1991.

RESUMO: São apresentados 14 casos de aneurismas e/ou dissecções da aorta torácica submetidos a correção cirúrgica com o emprego de hipotermia profunda e parada circulatória. Entre os 14 pacientes, seis eram portadores de aneurismas da aorta (um de aorta ascendente, três do arco aórtico, um de aorta ascendente + arco aórtico, um de aorta descendente) e oito eram portadores de dissecção aórtica. A hipotermia foi induzida gradualmente até atingir $18^{\circ} \mathrm{C}$ de temperatura nasofaríngea. $O$ período médio de parada circulatória foi de 35 minutos. Houve quatro óbitos hospitalares, sendo um por problemas hemorrágicos, um por arritmia ventricular pós IAM, um por insuficiência renal aguda já existente no pré-operatório e um por AVC e infecção pulmonar secundária. Dos 10 pacientes, dois apresentaram insuficiência respiratória com assistência ventilatória prolongada e quatro apresentaram distúrbios neurológicos transitórios, com recuperação total. A técnica de hipotermia profunda e parada circulatória mostrou-se como boa alternativa na abordagem cirúrgica de lesōes graves da aorta torácica.

DESCRITORES: aorta torácica, cirurgia com hipotermia profunda e parada circulatória.

\section{INTRODUÇÃO}

A introdução de técnicas cirúrgicas no tratamento das lesões da aorta torácica foi um fator decisivo na mudança da história natural dessas doenças, cuja má evoluçāo clínica já é bem estabelecida.

As doenças do arco aórtico, bem como algumas lesōes extensas da aorta torácica apresentam grandes dificuldades no tratamento cirúrgico, não só pela difícil exposição operatória, como também pela necessidade de interrupção sangüínea a órgãos nobres, principalmente ao sistema nervoso central.
Durante os últimos anos, inúmeras técnicas foram propostas para ultrapassar tais dificuldades, todas elas com alguns inconvenientes importantes. Neste espaço, o ressurgimento da hipotermia profunda veio trazer importante avanço no tratamento de aneurismas e dissecçōes da aorta torácica, por propiciar um excelente campo cirúrgico e possibilitar a realização de operações mais extensas em um único tempo. Apesar de apresentar algumas complicaçōes, podemos dizer que estas têm sido minoradas à medida em que se ganha experiência com a técnica. Neste trabalho, apresentamos nossa experiência com hipotermia profunda e parada circulatória no tratamento de 14 pacientes portadores de graves lesões da aorta torácica.

Trabalho realizado no Biocor Hospital de Doenças Cardiovasculares e na Santa Casa de Misericórdia de Belo Horizonte. Belo Horizonte, MG, Brasil. Apresentado ao 18: Congresso Nacional de Cirurgia Cardiaca. Rio de Janeiro, RJ, 5 e 6 de abril, 1991.

* Do Biocor Hospital de Doenças Cardiovasculares e da Santa Casa de Misericórdia de Belo Horizonte.

* Da Santa Casa de Misericórdia de Belo Horizonte.

Endereço para separatas: Bayard Gontijo Filho. Caixa Postal 106. 30161 Belo Horizonte, MG, Brasil. 
GONTIJO FILHO, B.; FANTINI, F. A.; BARBOSA, J. T.; SILVA, J. A. P.; CASTRO, M. F.; PEREDO, E. O. A.; PEDROSA, A. A.; GONÇALVES, F. D.; VRANDECIC, M. O. - Correçāo de doenças da aorta torácica com utilização de hipotermia profunda e parada circulatória. Rev. Bras. Cir. Cardiovasc., 6(1): 11-16, 1991.

\section{CASUISTICA E MÉTODOS}

Entre janeiro de 1980 e fevereiro de 1991, foram operados 110 pacientes portadores de aneurismas e dissecçōes da aorta torácica, nos Serviços de Cirurgia Cardiovascular da Santa Casa de Misericórdia de Belo Horizonte e Biocor Hospital de Doenças Cardiovasculares. Diversas técnicas cirúrgicas foram utilizadas nesses pacientes, que variaram fundamentalmente em funçāo da topografia da lesão e do quadro clínico do paciente.

A hipotermia profunda com parada circulatória foi empregada em 14 casos, nesse grupo. A idade dos pacientes variou de 27 a 75 anos (idade média $=54$ anos) havendo predominância do sexo $\mathrm{M}$ (oito casos). Conforme demonstra a Tabela 1, houve, nessa série, um predomínio discreto do número de dissecções da aorta sobre o de aneurismas. $O$ acometimento do arco aórtico foi a indicação principal do emprego da hipotermia profunda (nove casos); desses, quatro eram portadores de aneurismas e cinco de dissecçōes com origem no arco aórtico e/ou extensão retrógrada. Em um paciente portador de aneurisma gigante da aorta ascendente havia erosão completa do esterno, sem possibilidade de abordagem da lesão a não ser por parada circulatória.

Em dois pacientes, o procedimento foi utilizado para tratamento de lesōes da aorta descendente, sendo um paciente portador de dissecção aguda, no qual foi realizada a técnica de "tromba de elefante" e a outra, portadora de enorme aneurisma de toda a aorta torácica descendente, cujo acesso à parte proximal da aorta era praticamente impossivel sem uma interrupção do fluxo sangüíneo.

Finalmente, dois pacientes com dissecção aguda da aorta ascendente foram submetidos a períodos de parada circulatória para realização da anastomose aórtica distal ("open distal anastomosis").

TABELA 1

LESÕES AORTICAS CORRIGIDAS POR HIPOTERMIA PROFUNDA E PARADA CIRCULATORIA

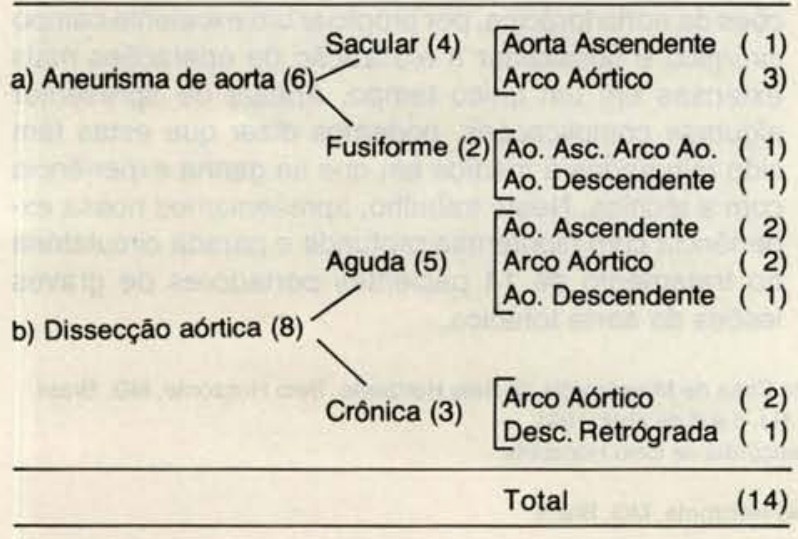

Quatro pacientes necessitaram procedimentos associados, além da correção da lesão aórtica, sendo a revascularização miocárdica realizada em três pacientes e a troca de válvula aórtica em um paciente.

\section{Técnica Cirúrgica}

A hipotermia profunda foi induzida em todos os pacientes apenas pela circulação extracorpórea com o oxigenador de bolhas, sem o uso de resfriamento de superfície. O circuito foi estabelecido entre o átrio direito (AD) e artéria femoral (AF) em 13 pacientes. Na paciente portadora de aneurisma de aorta descendente, operada através de toracotomia esquerda, a drenagem venosa foi obtida pela veia femoral e artéria pulmonar. No paciente em que foi realizada a técnica de "tromba de elefante", o retorno arterial foi transferido para a aorta ascendente, na fase de reaquecimento.

A hipotermia foi induzida progressivamente, até uma temperatura de $18^{\circ} \mathrm{C}$, sendo o tempo médio de esfriamento de 33 minutos. A parada cardiocirculatória com drenagem sangüínea ao reservatório variou de 16 a 56 minutos (tempo médio $=35$ minutos). $O$ tempo médio de reaquecimento foi de 48 minutos.

Durante o período de hipotermia, o equilibrio ácido básico foi abordado pelo método "alpha - stat" sem a correção do PH pela temperatura. Procuramos utilizar o mínimo de soluções glicosadas e o hematócrito foi mantido entre $20 \%-30 \%$. Antes da parada circulatória, foram administrados corticóides (500 mg de metilprednisolona) e barbitúricos (40 mg/kg de tiopental), para se conseguir melhor proteção cerebral.

Os vasos cerebrais não foram ocluídos, na maioria dos pacientes, porém a posição de Trendelenburg foi sempre empregada, antes de se incisar a aorta.

\section{RESULTADOS}

Houve um óbito na sala de operação e três pacientes faleceram no período pós-operatório imediato. Nesses quatro pacientes, apenas um dos óbitos pôde ser relacionado à hipotermia com parada circulatória. Tratava-se da paciente com aneurisma de aorta descendente, que apresentou acidente vascular cerebral importante no pós-operatório imediato, seguido de infecção pulmonar grave.

Outro paciente portador de dissecção aguda da aorta e insuficiência renal aguda pré-operatória persistiu com o problema no pós-operatório, sendo esta a causa de óbito. Uma paciente faleceu na sala de operaçōes. Tratava-se de paciente portadora de aneurisma gigante da aorta descendente, apresentava a aorta ascendente e o arco aórtico totalmente calcificados, não sendo possí- 
GONTIJO FILHO, B.; FANTINI, F. A.; BARBOSA, J. T.; SILVA, J. A. P.; CASTRO, M. F.; PEREDO, E. O. A.; PEDROSA, A. A.; GONÇALVES, F. D.; VRANDECIC, M. O. - Correção de doenças da aorta torácica com utilização de hipotermia profunda e parada circulatória. Rev. Bras. Cir. Cardiovasc., 6(1): 11-16, 1991.

vel coibir a hemorragia após a correção cirúrgica, vindo a falecer na sala de operaçōes. O outro paciente faleceu no pós-operatório imediato, após apresentar arritmia ventricular secundária a insuficiência coronária aguda.

Nos 10 pacientes restantes, a principal complicação pós-operatória foi a insuficiência respiratória, com assistência ventilatória prolongada em dois casos. Não observamos distúrbios de coagulação significativa nesse grupo. Distúrbios neurológicos discretos com recuperaçāo completa ocorreram em quatro pacientes; a hemodiálise foi empregada em uma paciente portadora de insuficiência renal crônica, em programa de diálise no pré-operatório.

Dois pacientes que apresentavam compressão extrínseca de artéria pulmonar e veia cava superior pelo aneurisma tiveram regressāo completa do quadro clínico, após a correção.

\section{DISCUSSÃO}

Apesar de relatos anteriores, foi o trabalho de GRIEPP et alii ${ }^{7}$, de 1975 , que maior estímulo trouxe para a utilização da hipotermia profunda no tratamento de aneurismas e dissecçōes aórticas. Nesse relato, foi apresentada uma sobrevida de três entre quatro pacientes submetidos a correção de lesōes do arco aórtico com parada cardiocirculatória. Posteriormente, vários outros relatos, com números crescentes de pacientes, foram publicados, atestando a importância dessa técnica $^{1}, 3,5,10,11$

Desde o início do seu emprego, duas têm sido as principais áreas de preocupação com relação a hipotermia profunda e parada circulatória: a efetividade da proteção do sistema nervoso central (SNC) e os distúrbios de coagulação.

A larga experiência com a hipotermia profunda no tratamento das cardiopatias congênitas demonstrou ser esse um método bastante eficaz na proteção do SNC, desde que o período de parada circulatória não ultrapasse 45 a 60 minutos, a $18^{\circ} \mathrm{C}$ (temperatura nasofaríngea) ${ }^{8}$. Em pacientes adultos, entretanto, não existem estudos que comprovem esse fato. Os resultados clínicos, porém, demonstram que essa correlação provavelmente verdadeira, também nessa faixa etária. SWEENEY et alii ${ }^{12}$ apresentam ótimos resultados pós-operatórios, com periodos mais curtos de parada circulatória à temperaturas de $20-24^{\circ} \mathrm{C}^{12}$. GRIEPP *, em uma atualização da experiência do Mount Sinai Hospital, detectou, entre 87 sobreviventes de um grupo de 96 pacientes submetidos a hipotermia profunda, apenas um caso de lesão neurológica permanente (104 minutos de parada

\footnotetext{
- Comunicação pessoal. 2. Simpósio de Doenças da Aorta. New York,
} USA, 1990. circulatória) e 14 casos de lesões transitórias. LANSMAN et alii ${ }^{9}$ aconselham baixar a temperatura a $15^{\circ} \mathrm{C}$ quando se prevê um tempo superior a 20 minutos de parada circulatória, ou quando existe o diagnóstico de dissecção aórtica com origem no arco aórtico.

Para se obter uma proteçāo eficaz no SNC com a hipotermia, é importante que o seu estabelecimento seja gradual assim como o reaquecimento. CONNOLY et alii ${ }^{2}$, em 1965, demonstraram a formação de áreas tissulares mal perfundidas em casos de esfriamento rápido e também a formação de microêmbolos em reaquecimento mal conduzido.

Em recente trabalho, FRIST et alii ${ }^{6}$ indicam o uso de perfusão do tronco braquiocefálico associada a hipotermia moderada como forma de se obter boa proteção do SNC, sem os inconvenientes da hipotermia profunda, baseadas na análise de 10 casos. Outros autores, entretanto, contestam, dizendo que os riscos da canulação de vasos cerebrais, a dificuldade de manutenção do fluxo sangüíneo cerebral, bem como as inúmeras variações anatômicas do suprimeiro sangüíneo do SNC tornariam o método menos vantajoso do que a hipotermia profunda.

O uso de barbitúricos antes da parada circulatória tem sido empregado pela maioria das equipes cirúrgicas também como forma de proteção do SNC. Sua ação principal seria a de produzir uma diminuição do metabolismo cerebral, como também a de exercer um papel protetor importante no período de reperfusāo contra a ação dos radicais livres ${ }^{4}$.

Em nossa casuística, apenas um paciente apresentou complicação neurológica grave, por provável embolia gasosa. Na nossa opinião, o ar retido na aorta ascendente durante a correção do aneurisma da aorta torácica descendente foi a causa do problema. Nessa situação, a utilização da posiçāo de trendelenburg e a troca do fluxo arterial da artéria femoral para a aorta ascendente na fase de reaquecimento poderiam evitar tal complicação.

Os efeitos da hipotermia profunda sobre o sistema de coagulação são bastante conhecidos. Há diminuição sérica do fator VII, aumento da atividade fibrinolítica, diminuição no número de plaquetas, além de um aumento da atividade antitrombina após o reaquecimento ${ }^{10}$. A soma desses fatores pode ser crítica, ao final da correção cirúrgica.

As próteses têxteis, mesmo com baixo nível de porosidade, podem ser permeáveis ao sangue, gerando conseqüências desastrosas. Tal fato tem sido evitado com métodos alternativos de pre-coagulação da prótese a altas temperaturas. No nosso Serviço, temos empregado, quase rotineiramente, os enxertos de pericárdio bovino, que, além de ótima qualidade para sutura, nāo apresentam nenhum grau de porosidade. Além disso, a utilização sistemática de fatores de coagulação após neutra- 
GONTIJO FILHO, B.; FANTINI, F. A.; BARBOSA, J. T.; SILVA, J. A. P.; CASTRO, M. F.; PEREDO, E. O. A.; PEDROSA, A. A.; GONÇALVES, F. D.; VRANDECIC, M. O. - Correção de doenças da aorta torácica com utilização de hipotermia profunda e parada circulatória. Rev. Bras. Cir. Cardiovasc., 6(1): 11-16, 1991.

lização da heparina praticamente elimina esse problema. Na nossa série, não observamos casos de coagulopatia significativa. Um paciente faleceu em consequeência de hemorragia, porém de causa cirúrgica.

Na nossa opiniâo, a técnica de hipotermia profunda com parada circulatória apresenta inúmeras aplicações clínicas no tratamento de doenças da aorta. A nossa confiança atual no procedimento provavelmente teria mudado nossa conduta em casos anteriormente abordados por outras técnicas.

As principais complicações inerentes à hipotermia profunda poderão ser melhor controladas através de um planejamento cirúrgico detalhado e coordenado, de forma a se conseguir o menor período de parada circulatória.

RBCCV 44205-126

CONTIJO FILHO, B.; FANTINI, F. A.; BARBOSA, J. T.; SILVA, J. A. P.; CASTRO, M. F.; PEREDO, E. O. A.; PEDROSA, A. A.; GONÇALVES, F. D.; VRANDECIC, M. O. - Surgical treatment of thoracic aortic lesions with deep hypothermia and circulatory arrest. Rev. Bras. Cir. Cardiovasc., 6(1): 11-16, 1991.

ABSTRACT: The authors report 14 cases of aneurysms and/or dissections of the thoracic aorta, treated with deep hypothermia and circulatory arrest. There were 6 aneurysms of the aorta ( 1 of the ascending aorta, 3 of the aortic arch, 1 of the ascending aorta and aortic arch, 1 of the descending aorta) and 8 cases of aortic dissections. The patients were gradually colled down to $18^{\circ} \mathrm{C}$ of nasopharingeal temperature. The mean circulatory arrest time was 35 minutes. There were 4 hospital deaths; 1 patient died in the operating room, from persistent surgical bleeding; 1 patient died from acute renal failure, which was already present in the pre-operative period; 1 patient died after an acute myocardial infarction followed by ventricular fibrillation; 1 patient died from pulmonary infection following a carebro-vascular accident. This was the only death directly related to the circulatory arrest technique. Among the 10 survivors, there were 2 patients who required prolonged ventilatory support and 4 minor neurological abnormalities with complete recovery. The technique of deep hypothermia and circulatory arrest proved to be a good alternative in the treatment of difficult aortic lesions.

DESCRIPTORS: thoracic aorta, surgery with deep hypothermia and circulatory arrest.

\section{REFERÊNCIAS BIBLIOGRÁFICAS}

1 ANTUNES, M. J.; COLSEN, P. R.; KINSLEY, R. H. Hypothermia and circulatory arrest for surgical resection of aortic arch aneurysms. H. Thorac. Cardiovasc. Surg., 86: 576-581, 1983.

2 CONNOLY, J. E.; ROY, A.; GUERNSEY, J. M.; STERMMER, E. A. - Bloodless surgery by means of profound hypothermia and circulatory arrest: effect of brain and heart. Ann. Thorac. Surg., 162: 724-737, 1965.

3 CRAWFORD, E. A.; SVENSSON, L. G.; CONSELLI, J. S.; SAFI, H. J.; HESS, K. R. - Surgical treatment of aneurysm and/or dissection of the ascending aorta, transverse aortic arch and transverse aortic arch: factors influencing survival in 717 patients. J. Thorac. Cardiovasc. Surg., 98: 659-674, 1989.

4 CREEPS Jr., J. T.; ALLMENDINGER, P.; ELLISON, L:; HUMPHREY, C.; PREISLLER, P.; LOW, H. - Hypothermia circulatory arrest in the treatment of thoracic aortic lesions. Ann. Thorac. Surg., 43:644-647, 1987.

5 ERGIN, M. A.; O'CONNOR, J.; GUINTO, R.; GRIEPP, R. A. - Experience with profound hypothermia and circu- latory arrest in the treatment of aneurysms of the aortic arch: aortic arch replacement for acute arch dissections. J. Thorac. Cardiovasc. Surg., 84: 649-655, 1982.

6 FRIST, W. H.; BALDWIN, J. C.; STARNES, V. A.; STINSON, E. B.; OYER, P. E.; MILLER, C.; JAMIESON, S. W.; MITCHELL, R. S.; SHUMWAY, N. E. - A reconsideration of cerebral perfusion in aortic arch replacement. Ann. Thorac. Surg., 42: 273-281, 1986.

7 GRIEPP, R. B.; STINSON, E. B.; HOLLINSGSWORTH, J. F.; BUCHLER, D. - Prosthetic replacement of the aortic arch. J. Thorac. Cardiovasc. Surg., 70: 1055-1063, 1975.

8 KIRKLIN, J. N.; \& BARRATT-BOYES, B. G. - Cardiac Surgery. New York, John Willey and Sons, 1986. p. 41.

9 LANSMAN, S. L.; RAISSI, S.; ERGIN, M. A.; GRIEPP, R. B. - Urgent operation for acute transverse aortic arch dissection. J. Thorac. Cardiovasc. Surg., 97: 334-341, 1989.

10 LIVESAY, J. J.; COOLEY, D. A.; REUL, G. T.; WALKER, W. E.; FRAZIER, O. H.; DUNCAN, J. M.; OTT, D. A. 
GONTIJO FILHO, B.; FANTINI, F. A.; BARBOSA, J. T.; SILVA, J. A. P.; CASTRO, M. F.; PEREDO, E. O. A.; PEDROSA, A. A.; GONÇALVES, F. D.; VRANDECIC, M. O. - Correçāo de doenças da aorta torácica com utilização de hipotermia profunda e parada circulatória. Rev. Bras. Cir. Cardiovasc., 6(1): 11-16, 1991.

- Ressection of aortic arch aneurysms: a comparison of hypothermia techniques in 60 patients. Ann. Thorac. Surg., 36: 19-28, 1983.

11 MAHFOOF, S.; QAZI, A.; GARCIA, J.; MISPIRETA, L.; CORSO, P.; SMYTH, N. - Management of aortic arch aneurysms using profound hypothermia and circulatory arrest. Ann. Thorac. Surg., 39: 412-417, 1985.

12 SWEENEY, M. S.; COOLEY, D. A.; REUL, G. T.; OTT, D. A.; DUNCAN, J. M. - Hypothermic circulatory arrest for cardiovascular lesions: technical considerations and results. Ann. Thorac. Surg., 40: 498-503, 1985.

\section{Discussão}

\section{DR. ISEU AFFONSO DA COSTA Curitiba, $P R$}

O trabalho do Dr. Gontijo e colaboradores representa uma importante contribuição prática para estimular o uso da hipotermia profunda no tratamento de lesões da aorta torácica, em nosso meio. Os resultados apresentados, tratando-se de uma área tão difícil da terapêutica cirúrgica cardiovascular, são animadores. A experiência do nosso grupo, ainda que apresentando incidência apreciável de complicaçōes próprias do tipo das lesōes tratadas, aponta na mesma direção. Sem dúvida, é necessário que troquemos informaçōes sobre as dificuldades encontradas e, com o aumento do número de casos operados pelos vários grupos, cheguemos a conclusōes confiáveis. Procuramos estudar os resultados do Dr. Gontijo, face aos dados de Kirklin sobre a hipotermia profunda. Assim, vemos que o tempo de parada circulatória total variou entre 16 e 56 minutos. Com 16 minutos, estamos dentro de uma faixa bem segura, enquanto com 56, e probabilidade de uma parada total segura, é de apenas cerca de $30 \%$. Seria interessante verificar, entre os pacientes da série apresentada que mostraram lesões neurológicas discretas, qual a duração de parada circulatória total. A projeção de Kirklin é baseada no uso de técnica em crianças mas, como dizem o Dr. Gontijo e colaboradores, em seu texto, parece não haver dados correspondentes em pacientes adultos, e não é seguro afirmar quanto se pode extrapolar os dados. Outro pormenor relevante é o tempo de "equilibrio térmico" decorrido desde o momento em que se atinge a temperatura de $18^{\circ} \mathrm{C}$ no nasofaringe e a parada circulatória. Temos por rotina observar um intervalo de $30 \mathrm{minu}-$ tos, após atingida a temperatura desejada e a parada circulatória, objetivando mais perfeita homogeneização da temperatura dos órgāos, especialmente o encéfalo. Nas crianças, com as quais a experiência cirúrgica é bem maior, usamos 15 minutos para a homogeneização técnica. Aparentemente, essa não é o rotina do $\mathrm{Dr}$. Gontijo e seu grupo. Como ressaltamos no início deste comentário, é necessário observar-se maior número de casos, para se obterem dados mais confiáveis. A série apresentada, embora seja significativa, face à gravidade das lesões e à complexidade das operaçōes e representar uma experiência cirúrgica respeitável, mostra números dificilmente analisáveis, do ponto de vista estatístico. Cumprimentamos o Dr. Gontijo e seus associados, pela contribuição oferecida ao conhecimento do tema em nosso meio e os estimulamos à apresentação futura da evolução de sua experiência, nesta difícil área de cirurgia cardiovascular.

\section{DR. RAUL CORREAA RABELO Belo Horizonte, MG}

Gostaríamos de cumprimentar os autores do trabaIho em questão, pelos bons resultados obtidos. Em nosso Serviço no Instituto do Coração do Hospital Madre Teresa (Belo Horizonte-MG), temos empregado a hipotermia profunda com parada circulatória total em todos os casos de dissecção aguda da aorta, nos aneurismas que interessam ao arco aórtico e, eventualmente, em outras situações, como na tromboembolectomia pulmonar, com circulação extracorpórea (CEC). Nossa técnica é simples: resfriamento gradativo sistêmico até $18^{\circ} \mathrm{C}$ de temperatura esofágica, sem uso de corticóides ou barbitúricos, posição de Trendelemburg, exsangüinação do paciente, mantendo lenta circulação fechada entre o oxigenador e o reservatório de cardiotomia. A reintrodução do sangue é feita pela via venosa, através do circuito das cavas, e a transferência da cânula arterial para a aorta ascendente ao ser reinstalada a CEC. Desde a publicação de Fabiani et alii (1990), temos abordado as dissecçōes agudas da aorta com emprego da cola biológica (Cirumédica) aquecida a $37^{\circ} \mathrm{C}$, e colocada entre as lâminas dissecadas da aorta, após exaustiva secagem local. Para perfeita aposição das lâminas dissecadas, usamos prodigamente a cola (média de 6 tubos/paciente) e temos utilizado, com sucesso, clipes de aço para papel esterelizados, que exercem excelente pressão uniforme sobre os tecidos a serem colados. Após sete a dez minutos, obtemos excelente consistência, firmeza e resistência dos tecidos, permitindo sutura segura por chuleio simples, sem necessidade de interposição de próteses vasculares. Foram operados cinco pacientes desde dezembro de 1990, com dissecção aguda da aorta, sendo um caso do tipo II de De Bakey e quatro do tipo I de De Bakey, todos com algum grau de insuficiência aórtica. Em todos os casos, foi utilizada a técnica descrita, sem interposição de próteve vascular e sem necessidade de substituição valvar aórtica. Ocorreu um óbito na série, sem relação com as técnicas descritas, pois resultou de AVC pré-existente, conseqüente a dissecçăo e semi-oclusão do tronco braquiocefálico. Caso houvesse necessidade de uso de próteses vasculares, nossa opção seria pelas próteses de pericárdio bovino, pelas razōes declinadas pelos autores do trabalho em discussão. Concluímos que a associação da hipotermia profunda com parada circulatória total, somada à cola bioló- 
GONTIJO FIL.HO, B.; FANTINI, F. A.; BARBOSA, J. T.; SILVA, J. A. P.; CASTRO, M. F.; PEREDO, E. O. A.; PEDROSA, A. A.; GONÇALVES, F. D.; VRANDECIC, M. O. - Correçăo de doenças da aorta torácica com utilização de hipotermia profunda e parada circulatória. Rev. Bras. Cir. Cardiovasc., 6(1): 11-16, 1991.

gica, descortina amplo horizonte no tratamento das lesōes da aorta.

\section{DR. GONTIJO FILHO \\ (Encerrando)}

Agradeço os comentários elogiosos dos colegas e também os cumprimentos pelos resultados obtidos com a técnica de hipotermia profunda e parada circulatória. Com respeito à cola biológica ressaltada pelo Dr. Raul, nós também a empregamos, porém nunca como tratamento isolado da dissecção aórtica. Na nossa experiência, a cola é bastante útil na sutura proximal da aorta, ao passo que não demonstra o mesmo efeito no segmento distal e no arco aórtico. O ponto levantado pelo Dr. Iseu, a respeito do período seguro de parada circulatória sem acarretar danos ao sistema nervoso central, é controvertido. Alguns autores com maior experiência neste campo consideram aceitáveis períodos de até 60 minutos de parada circulatória com hipotermia de $18^{\circ} \mathrm{C}$. Entretanto, caso exista possibilidade deste tempo se prolongar acima de 60 minutos, achamos conveniente proceder como o Dr. Griepp, e esfriar até $14^{\circ} \mathrm{C}$. As lesōes neurológicas por nós encontradas foram fugazes, sem seqüelas, e nāo se relacionaram ao tempo de parada circulatória. Acredito que a hipotermia induzida de maneira gradual, com controle rigoroso do equilibrio ácidobásico, dispensa o período de homogeneização proposto pelo Dr. Iseu. Por outro lado, o resfriamento externo do crânio é útil e deve ser empregado, com intuito de se manter a temperatura cortical. Apesar dos mecanismos fisiológicos da hipotermia no homem năo estarem totalmente definidos, estou certo de se tratar de um método muito importante no tratamento de lesōes da aorta torácica. 